Supplement of Web Ecol., 18, 143-151, 2018

https://doi.org/10.5194/we-18-143-2018-supplement

(C) Author(s) 2018. This work is distributed under

the Creative Commons Attribution 4.0 License.

(c) (1)

Supplement of

\title{
The older the richer: significant increase in breeding bird diversity along an age gradient of different coppiced woods
}

Lorenzo Mentil et al.

Correspondence to: Corrado Battisti (c.battisti@ cittametropolitanaroma.gov.it)

The copyright of individual parts of the supplement might differ from the CC BY 4.0 License. 


\section{Supplementary materials 1.}

Rarefaction curves with 95\% confidence intervals calculated after 9,999 bootstraps. Cumulative percentage of species (y-axis) along progressive research effort (sampling points: $x$-axis). The curves were fitted using PAST 3.0 statistical software. Ch: Chestnuts; Ok: Turkey oaks; Be: Beech forests.

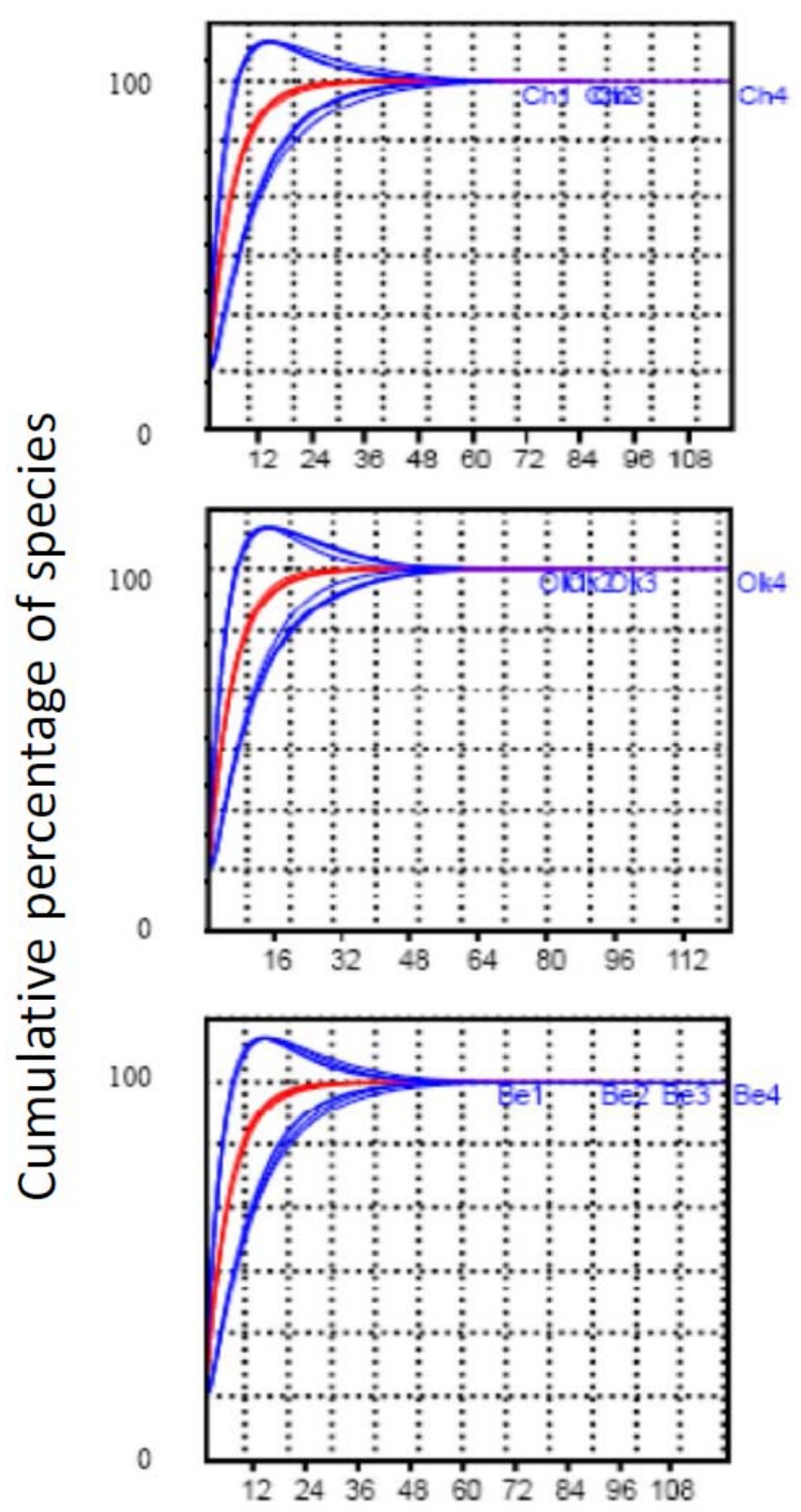

Progressive research effort 


\section{Supplementary materials 2.}

List of species observed in the study area. Forest species (i.e. generalist or specialized species inhabiting forest habitats) are indicated (*). See text for further details.

\begin{tabular}{|c|c|c|}
\hline Species & & $\begin{array}{c}\text { Forest } \\
\text { species }\end{array}$ \\
\hline Sparrowhawk & Accipiter nisus & \\
\hline Wood Pigeon & Columba palumbus & $*$ \\
\hline Turtle Dove & Streptopelia turtur & $*$ \\
\hline Hoopoe & Upupa epops & $*$ \\
\hline Cuckoo & Cuculus canorus & $*$ \\
\hline Green Woodpecker & Picus viridis & $*$ \\
\hline Great Spotted Woodpecker & Dendrocopos major & $*$ \\
\hline White-backed Woodpecker & Dendrocopos minor & $*$ \\
\hline Wryneck & Jynx torquilla & $*$ \\
\hline Blackbird & Turdus merula & $*$ \\
\hline Sardinian Warber & Sylvia melanocephala & \\
\hline Blackcap & Sylvia atricapilla & $*$ \\
\hline Chiffchaff & Phylloscopus collybita & $*$ \\
\hline Firecrest & Regulus ignicapilla & $*$ \\
\hline Wren & Troglodytes troglodytes & $*$ \\
\hline Robin & Erithacus rubecula & $*$ \\
\hline Spotted Flycatcher & Muscicapa striata & $*$ \\
\hline Great Tit & Parus major & $*$ \\
\hline Blue Tit & Cyanistes caeruleus & $*$ \\
\hline Marsh Tit & Poecile palustris & $*$ \\
\hline Long-tailed Tit & Aegithalos caudatus & $*$ \\
\hline Nuthatch & Sitta europaea & $*$ \\
\hline Wallcreeper & Certhia brachydactyla & $*$ \\
\hline Golden Oriole & Oriolus oriolus & $*$ \\
\hline Starling & Sturnus vulgaris & $*$ \\
\hline Jay & Garrulus glandarius & $*$ \\
\hline Chaffinch & Fringilla coelebs & $*$ \\
\hline Greenfinch & Chloris chloris & \\
\hline Serin & Serinus serinus & \\
\hline Subalpine Warbler & Sylvia cantillans & \\
\hline
\end{tabular}




\section{Supplementary materials 3}

Species and relative frequencies for each forest habitat (Ch, chestnut; Oa, Turkey oak; Be, beech woods) in the four age classes (from 1 to 4 ) considered. $\mathrm{N}=$ total number of records. Empty spaces correspond to species not present at specific sampling sites.

\begin{tabular}{|c|c|c|c|c|c|c|c|c|c|c|c|c|}
\hline species & Ch1 & Ch2 & Ch3 & Ch4 & Oa1 & $\mathrm{Oa} 2$ & Oa3 & $\mathrm{Oa} 4$ & $\mathrm{Be} 1$ & Be2 & $\mathrm{Be} 3$ & $\mathrm{Be} 4$ \\
\hline Accipiter nisus & & & & 0.010 & & & & & & & & 0.009 \\
\hline Columba palumbus & & 0.048 & 0.020 & 0.029 & & 0.068 & 0.072 & 0.036 & 0.027 & 0.035 & 0.045 & 0.053 \\
\hline Streptopelia turtur & & 0.016 & 0.020 & 0.019 & & 0.017 & 0.029 & 0.007 & 0.027 & 0.018 & 0.022 & 0.026 \\
\hline Upupa epops & 0.045 & & 0.020 & 0.010 & 0.021 & & & 0.015 & & & & \\
\hline Cuculus canorus & & & & 0.010 & & & 0.014 & & & & & \\
\hline Picus viridis & & & & 0.019 & & & 0.014 & 0.029 & & 0.035 & 0.045 & 0.044 \\
\hline Dendrocopos major & & & & 0.010 & & & 0.029 & 0.029 & 0.027 & 0.018 & 0.011 & 0.018 \\
\hline Dendrocopos minor & & & 0.020 & & & & 0.014 & 0.015 & & & & 0.009 \\
\hline Jynx torquilla & & & & 0.019 & & & 0.014 & 0.022 & 0.027 & 0.035 & 0.022 & 0.026 \\
\hline Turdus merula & 0.136 & 0.194 & 0.082 & 0.067 & 0.043 & 0.102 & 0.101 & 0.058 & 0.108 & 0.123 & 0.067 & 0.053 \\
\hline Sylvia melanocephala & & 0.048 & & & 0.064 & 0.017 & & & & & & \\
\hline Sylvia atricapilla & 0.045 & 0.048 & 0.061 & 0.048 & 0.064 & 0.068 & 0.072 & 0.058 & & 0.035 & 0.045 & 0.044 \\
\hline Phylloscopus collybita & 0.136 & 0.032 & 0.082 & 0.048 & 0.064 & 0.034 & 0.014 & 0.036 & 0.081 & 0.018 & 0.045 & 0.053 \\
\hline Regulus ignicapilla & 0.023 & 0.032 & 0.061 & 0.048 & 0.021 & 0.034 & 0.029 & 0.022 & & 0.053 & 0.045 & 0.044 \\
\hline Troglodytes troglodytes & 0.023 & 0.065 & 0.020 & 0.067 & & 0.034 & 0.072 & 0.066 & & 0.053 & 0.034 & 0.061 \\
\hline Erithacus rubecula & 0.091 & 0.161 & 0.061 & 0.077 & 0.106 & 0.136 & 0.130 & 0.073 & 0.027 & 0.053 & 0.045 & 0.053 \\
\hline Muscicapa striata & 0.045 & 0.032 & 0.020 & 0.038 & 0.021 & 0.017 & & 0.022 & 0.027 & 0.018 & 0.022 & 0.018 \\
\hline Parus major & 0.068 & 0.081 & 0.122 & 0.096 & 0.064 & 0.102 & 0.087 & 0.058 & 0.135 & 0.105 & 0.101 & 0.096 \\
\hline Cyanistes caeruleus & 0.091 & 0.081 & 0.122 & 0.087 & 0.085 & 0.102 & 0.087 & 0.080 & 0.108 & 0.105 & 0.079 & 0.088 \\
\hline Poecile palustris & 0.045 & & 0.041 & & & 0.034 & & & & 0.035 & & \\
\hline Aegithalos caudatus & & & 0.061 & 0.067 & 0.106 & 0.068 & 0.072 & 0.044 & 0.081 & 0.035 & 0.079 & 0.061 \\
\hline Sitta europaea & & & & 0.029 & & & & 0.066 & & 0.053 & 0.067 & 0.070 \\
\hline Certhia brachydactyla & & 0.016 & 0.041 & 0.048 & 0.043 & & 0.043 & 0.044 & 0.081 & 0.070 & 0.056 & 0.053 \\
\hline Oriolus oriolus & & 0.032 & 0.020 & & & 0.017 & 0.014 & 0.007 & & & & 0.009 \\
\hline Sturnus vulgaris & & & & 0.058 & & & & 0.102 & & & 0.022 & \\
\hline Garrulus glandarius & 0.068 & 0.081 & 0.041 & 0.019 & 0.128 & 0.119 & 0.072 & 0.029 & 0.054 & 0.035 & 0.056 & 0.035 \\
\hline Fringilla coelebs & 0.136 & 0.032 & 0.082 & 0.077 & 0.106 & 0.034 & 0.014 & 0.080 & 0.189 & 0.070 & 0.090 & 0.079 \\
\hline Chloris chloris & 0.045 & & & & & & & & & & & \\
\hline Serinus serinus & & & & & 0.021 & & & & & & & \\
\hline Sylvia cantillans & & & & & 0.043 & & & & & & & \\
\hline $\mathrm{N}$ & 44 & 62 & 49 & 104 & 47 & 59 & 69 & 137 & 37 & 57 & 89 & 114 \\
\hline
\end{tabular}

